\title{
Methylation of DLEC1 Promoter Is a Predictor for Recurrence in Chinese Patients with Gastric Cancer
}

\author{
Xiaobing Ye, ${ }^{1,2}$ Gang Feng, ${ }^{3}$ Nanlin Jiao, ${ }^{4}$ Chun Pu, ${ }^{3}$ Guohai Zhao, ${ }^{5}$ and Guoping Sun ${ }^{1}$ \\ ${ }^{1}$ Department of Medical Oncology, The First Affiliated Hospital of Anhui Medical University, Hefei, Anhui 230001, China \\ ${ }^{2}$ Department of Medical Oncology, The First Affiliated Hospital of Wannan Medical College, Wuhu, Anhui 241001, China \\ ${ }^{3}$ Clinical Genetics Laboratory, The First Affiliated Hospital of Wannan Medical College, Wuhu, Anhui 241001, China \\ ${ }^{4}$ Department of Pathology, The First Affiliated Hospital of Wannan Medical College, Wuhu, Anhui 241001, China \\ ${ }^{5}$ Department of Surgery, The First Affiliated Hospital of Wannan Medical College, Wuhu, Anhui 241001, China
}

Correspondence should be addressed to Guoping Sun; prguoping@126.com

Received 7 July 2014; Revised 19 November 2014; Accepted 24 November 2014; Published 9 December 2014

Academic Editor: Valeria Barresi

Copyright (C) 2014 Xiaobing Ye et al. This is an open access article distributed under the Creative Commons Attribution License, which permits unrestricted use, distribution, and reproduction in any medium, provided the original work is properly cited.

\begin{abstract}
Purpose. To investigate promoter methylation in the deleted in lung and esophageal cancer 1 (DLEC1) gene in Chinese patients with gastric cancer. Methods. A total of 227 patients with gastric cancer were enrolled. The methylations of the promoter regions of DLEC1 and ACTB were determined using quantitative methylation-specific PCR. The DLEC1 methylation was compared to the clinicopathological variables of gastric cancer. Results. DLEC1 methylation was not associated with the clinicopathological variables of gastric cancer. Patients with DLEC1-hypermethylated gastric cancer had significantly higher recurrence rate than those with DLEC1-hypomethylated gastric cancer $(P=0.025$; hazard ratio $=2.43)$. Conclusions. Methylation of DELC1 promoter may be a valuable predictor for recurrence in Chinese patients with gastric cancer.
\end{abstract}

\section{Introduction}

Gastric cancer is one of the most common malignancies and remains an important cause of mortality worldwide, especially in Asia $[1,2]$. The combination of surgical resection and adjuvant chemo- or radiotherapy has provided a significant improvement for the survival of patients with localized gastric cancer [3]. However, about $80 \%$ of the patients die within a short period of time from recurrence after curative surgery [4]. Therefore, early detection of recurrence is important for evaluating the treatment outcome and choosing the most effective management in patients with gastric cancer.

Contrast enhanced computed tomography (CT) is the most frequently used imaging modality for the detection of gastric cancer recurrence [5]. However, CT cannot reflect the presence and viability of cancer recurrence precisely because its diagnostic ability is dependent only on morphological changes of the involved organs and distorted anatomical structures [6]. Recently, integrated positron emission tomography (PET) with CT (PET-CT) for detection of gastric cancer recurrence after surgical resection has been reported [7-9].

Compared to more expensive imaging methods, analyses of tumor biomarkers have no risk of radiation exposure, are easily available, and are more cost effective. Accordingly, studies on tumor molecular markers in prognosis of gastric cancer are relevant. Many studies indicate that promoter $\mathrm{CpG}$ island hypermethylation is closely associated with inactivation of tumor suppressor genes in human cancers. Furthermore, all types of human cancer display promoter CpG island hypermethylation, although there are variations in the prevalence of $\mathrm{CpG}$ island hypermethylation among tumor types $[10,11]$. The stomach is one of the organs where aberrant $\mathrm{CpG}$ island hypermethylation occurs frequently during cancer development [12]. Many genes have been characterized to be inactivated by hypermethylation of their promoter $\mathrm{CpG}$ islands in gastric cancer [13].

Deleted in lung and esophageal cancer 1 (DLEC1) is a tumor-suppressor gene which suppresses tumor growth or reduces the invasiveness of cancer cells and promoter 
hypermethylation has been shown to be responsible for the silencing of DLEC1 in ovarian cancer and nasopharyngeal carcinoma [14]. Furthermore, promoter hypermethylation of DLEC1 has also been found in gastric cancer [15]. Ying et al. demonstrated that DLEC1 was downregulated or silenced in most gastric cell lines due to promoter methylation, whereas it was broadly expressed in normal stomach tissues [16].

The purpose of our study is to investigate the relationship of DLEC1 methylation with clinicopathologic variables and determine whether DLEC1 methylation has any prognostic significance in patients with gastric cancer.

\section{Materials and Methods}

2.1. Patients. The study group included gastric cancer patients who had undergone radical surgical resection (D2) from Jun 2008 to Jun 2010. All tissues were fixed in 10\% neutralized formalin, embedded in paraffin, cut into $4 \mu \mathrm{m}$ sections, and stained with hematoxylin and eosin (H\&E) in order to confirm the histological diagnosis and microscopic characteristics of the specimens. The staging for each gastric cancer was evaluated according to the Union for International Cancer Control system, which indicates the extent of tumor spread [17]. Histological architecture was defined using the Lauren classification [18]. The tumor size, depth of invasion, lymphatic and venous invasion, and lymph node metastasis of tumors were also determined.

No patients were treated with chemotherapy, radiotherapy, and adjuvant treatment prior to surgery. All patients except stage I patients were also treated with standard adjuvant chemotherapy of modified FOLFOX6 regimen.

Follow-up information about the postoperative clinical course of patients was available from outpatient medical records or telephone calls. Recurrence-free survival (RFS) was defined at the time of surgery to tumor recurrence. The end date of the follow-up study for conducting the analysis was Jun 2014. The study protocol was approved by the First Affiliated Hospital of Wanan Medical College and the First Affiliated Hospital of Anhui Medical University.

2.2. DNA Extraction and Bisulfite Treatment. Ten sections of $10 \mathrm{~mm}$ thickness of paraffin-embedded tissues were used for DNA extraction. The paraffin was removed from the tissue by rinsing in xylene and genomic DNA was isolated using a QIAamp tissue kit (Qiagen, Valencia, CA, USA). DNAs were stored at $-80^{\circ} \mathrm{C}$ before analysis. DNA $(1 \mu \mathrm{g})$ was treated with bisulfite to convert unmethylated cytosines to uracils using the EZ DNA Methylation-Gold Kit (Zymo Research Corporation, Irvine CA, USA) according to the manufacturer's protocol. After treatment, DNAs were stored at $-80^{\circ} \mathrm{C}$ until being used.

2.3. Quantitative Methylation-Specific PCR. The methylations of promoter were determined using quantitative methylation-specific PCR. The primers and probe for DLEC1 were $5^{\prime}$-TTT CGT TGC GTA TTT AAG ATA TTT C-3',
$5^{\prime}$-CGT AAC GCT CAT TCT CGC TAC C-3', and 6-FAM$5^{\prime}$-TAA TCA AAC TTA CGC TCA CTT CGT CGC CG$3^{\prime}$-6-TAMRA. The primers and probe for ACTB were $5^{\prime}$ TGG TGA TGG AGG AGG TTT AGT AAG T-3', $5^{\prime}$-AAC CAA TAA AAC CTA CTC CTC CCT TAA-3', and 6-FAM$5^{\prime}$-ACC ACC ACC CAA CAC ACA ATA ACA AAC ACA$3^{\prime}$-6-TAMRA. Amplification reactions were carried out in triplicate in a final volume of $20 \mu \mathrm{L}$ that contained $3 \mu \mathrm{L}$ of bisulfite-modified DNA; $600 \mathrm{nM}$ concentrations of forward and reverse primers; $200 \mathrm{nM}$ probe; $0.6 \mathrm{U}$ of platinum Taq polymerase (Invitrogen, Frederick, MD); $200 \mathrm{mM}$ concentrations each of dATP, dCTP, dGTP, and dTTP; and $6.7 \mathrm{mM}$ $\mathrm{MgCl}_{2}$. Amplifications were carried out using the following program: $95^{\circ} \mathrm{C}$ for 3 minutes, followed by 40 cycles at $95^{\circ} \mathrm{C}$ for 15 seconds, and $60^{\circ} \mathrm{C}$ for 1 minute. Amplification reactions were carried out in 384-well plates in Roche LightCycler 480II (Roche Applied Science) and were analyzed by LightCycler 480 software (version 1.3). Each plate included patient DNA samples, positive (in vitro methylated leukocyte DNA) and negative (normal leukocyte DNA) controls, and water blanks. A standard curve was generated using serial dilutions of CpGenome Universal Methylated DNA (Chemicon, Temecula, CA). DLEC1 methylation was defined as a ratio of methylation specific PCR-amplified DLEC1 to ACTB and then multiplied by 100 for easier tabulation.

2.4. Statistical Analyses. DLEC1 methylations were expressed as mean \pm SD. The associations between DLEC1 methylation and the clinicopathological variables were assessed by MannWhitney $U$ test. Receiver operator curves (ROC) were used to compare the ability to identify patients with recurrence by DLEC1 methylation. RFS was generated using KaplanMeier estimates, and the difference between curves was evaluated with the Log-rank test. Hazard ratios (HRs) and 95\% confidence intervals (CIs) computed from multivariate analysis were used to investigate the relationship between RFS and variables. Differences were considered significant at a level of $P<0.05$. All statistical analyses were performed using the SPSS 13.0 statistical package.

\section{Results}

A total of 227 patients with gastric cancer were enrolled into the study. There were 157 males and 70 females with age of $63.15 \pm 12.02$ years (range $35-86$ ). The associations between DLEC1 methylation and clinicopathological variables were shown in Table 1. DLEC1 methylation was not associated with age and gender $(P=0.392, P=0.421)$. In addition, $D L E C 1$ methylation did not correlate with tumor size $(P=0.243)$, depth of invasion $(P=0.066)$, lymphatic invasion $(P=$ $0.102)$, venous invasion $(P=0.074)$, TNM staging $(P=$ $0.063)$, Lauren classification $(P=0.050)$, and lymph node metastasis $(P=0.089)$.

Among 148 patients without lymph node metastasis, $67(45.3 \%)$ patients were found to have a recurrence after surgery. Table 2 described the sensitivity and specificity of clinicopathological factors for recurrence. DLEC1 methylation was significantly higher in patients with recurrence, as 
TABLE 1: Correlation between clinicopathological variables and DLEC1 methylation.

\begin{tabular}{lccc}
\hline $\begin{array}{l}\text { Clinicopathological } \\
\text { variables }\end{array}$ & Number & $\begin{array}{c}\text { DLEC1 } \\
\text { methylation }\end{array}$ & $P$ value \\
\hline Age (years) & & & 0.392 \\
$\quad \leq 65$ & 117 & $49.27 \pm 28.98$ & \\
$\quad>65$ & 110 & $48.54 \pm 36.04$ & \\
Gender & & & 0.421 \\
$\quad$ Male & 157 & $48.90 \pm 29.73$ & \\
$\quad$ Female & 70 & $48.77 \pm 38.29$ & \\
Tumor size (cm) & & & 0.243 \\
$\quad<4$ & 118 & $46.56 \pm 30.78$ & \\
$\quad$ (1) & 109 & $51.44 \pm 32.06$ & \\
Depth of invasion & & & 0.066 \\
$\quad$ Tis-1 & 116 & $45.05 \pm 31.05$ & \\
$\quad$ T2-4 & 111 & $52.94 \pm 33.43$ & \\
Lymphatic invasion & & & 0.102 \\
$\quad$ Positive & 82 & $53.88 \pm 37.61$ & \\
$\quad$ Negative & 145 & $46.09 \pm 28.02$ & \\
Venous invasion & & & 0.074 \\
$\quad$ Positive & 33 & $58.89 \pm 35.19$ & \\
$\quad$ Negative & 194 & $47.21 \pm 31.57$ & \\
UICC TNM staging & & & \\
$\quad$ 0-I & 41 & $42.01 \pm 24.49$ & \\
$\quad$ II-IV & 186 & $50.43 \pm 33.21$ & \\
Lauren classification & & & \\
$\quad$ Intestinal type & 122 & $44.12 \pm 29.50$ & \\
$\quad$ Diffuse type & 105 & $54.48 \pm 35.04$ & \\
Lymph node & & & \\
metastasis & & & \\
$\quad$ Positive & & & \\
$\quad$ Negative & & & \\
\hline
\end{tabular}

compared with that in patients without recurrence $(P=$ 0.012 , Figure 1). ROC analyses of DLEC1 methylation in patients with and without recurrence are shown in Figure 2. In this study population, the best cut-off point for DLEC1 methylation was 35.10. DLEC1 methylation of 35.10 demonstrated a sensitivity and specificity of $70.1 \%$ and $51.9 \%$, respectively, for recurrence $(\mathrm{ROC} \mathrm{AUC}=0.648 ; 95 \% \mathrm{CI}$, 0.560-0.736).

Figure 3 showed that Kaplan-Meier analysis of RFS based on DLEC1 methylation using 35.10 as the optimal threshold. DLEC1 methylation was associated with RFS in the evaluated cohort $(P=0.028)$. A multivariate Cox proportional hazards model using variables associated with RFS in our study indicated that depth of invasion, lymphatic invasion, venous invasion, TNM staging, Lauren classification, and lymph node metastasis $(P<0.05)$, but not age, gender, or tumor size $(P>0.05)$, were independent predicted factors for recurrence in gastric cancer. Although the impact of DLEC1

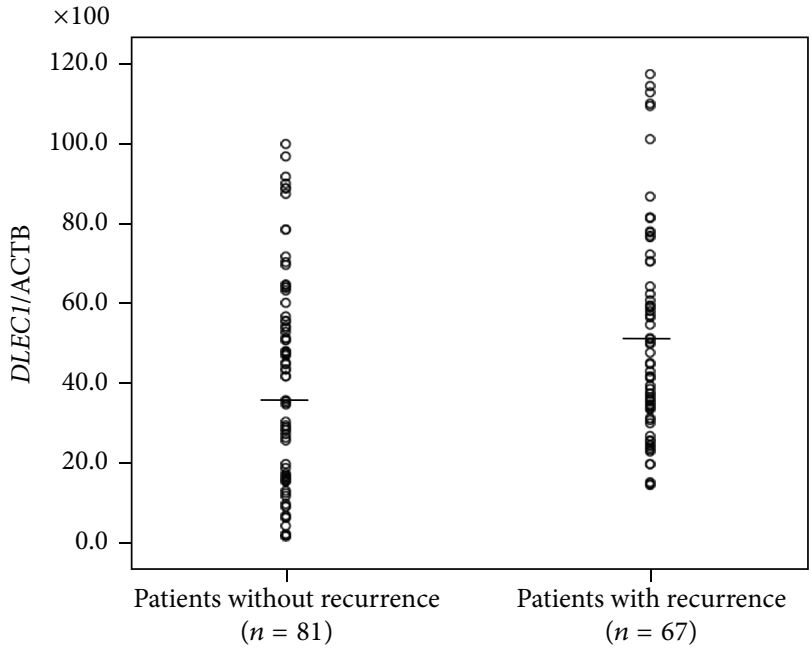

FIGURE 1: Scatter plots showing methylation levels of DLEC1 in gastric tumor separated by recurrence. Calculation of DLEC1 to ACTB ratio was based on the fluorescence emission intensity values for both genes obtained by quantitative methylation-specific PCR analysis. The obtained ratios were multiplied by 100 for easier tabulation.

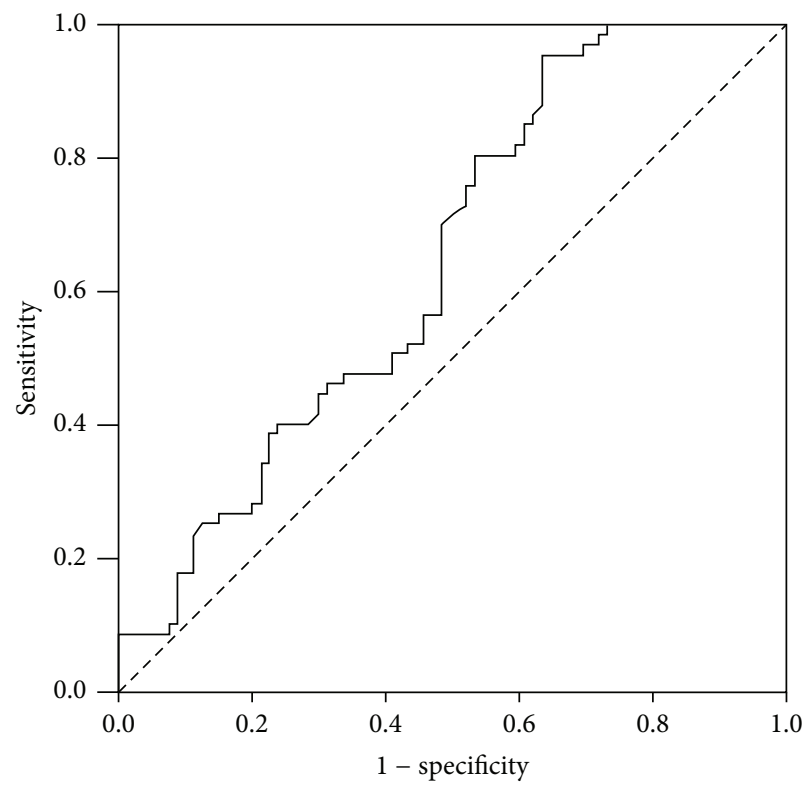

FIGURE 2: Receiver operating characteristic (ROC) curve of DLEC1 methylation in predicting recurrence in patient without lymph node metastasis after surgery. The area under the curve (AUC) was 0.648 (0.560-0.736). The best cutoff value was 35.10 (sensitivity, $70.1 \%$; specificity, $51.9 \%)$.

methylation on RFS was less evident than depth of invasion, lymphatic invasion, venous invasion, Lauren classification, and lymph node metastasis, the risk of recurrence in patients with higher DLEC1 methylation was still 2.43 times higher than those with lower DLEC1 methylation $(P=0.025)$ (Table 3). 
TABLE 2: The sensitivity and specificity of clinicopathological factors for recurrence.

\begin{tabular}{|c|c|c|c|c|c|c|}
\hline Factors & & Recurrence & Sensitivity & Specificity & PPV & NPV \\
\hline \multicolumn{7}{|l|}{ Tumor size $(\mathrm{cm})$} \\
\hline$<4$ & 85 & 38 & \multirow{2}{*}{$43.3 \%$} & \multirow{2}{*}{$58.0 \%$} & \multirow{2}{*}{$46.0 \%$} & \multirow{2}{*}{$55.3 \%$} \\
\hline$\geq 4$ & 63 & 29 & & & & \\
\hline \multicolumn{7}{|l|}{ Depth of invasion } \\
\hline Tis-1 & 102 & 27 & \multirow{2}{*}{$59.7 \%$} & \multirow{2}{*}{$92.5 \%$} & \multirow{2}{*}{$86.9 \%$} & \multirow{2}{*}{$73.5 \%$} \\
\hline $\mathrm{T} 2-4$ & 46 & 40 & & & & \\
\hline \multicolumn{7}{|l|}{ Lymphatic invasion } \\
\hline Positive & 18 & 16 & \multirow{2}{*}{$23.9 \%$} & \multirow{2}{*}{$97.5 \%$} & \multirow{2}{*}{$88.9 \%$} & \multirow{2}{*}{$60.8 \%$} \\
\hline Negative & 130 & 51 & & & & \\
\hline \multicolumn{7}{|l|}{ Venous invasion } \\
\hline Positive & 9 & 7 & \multirow{2}{*}{$10.4 \%$} & \multirow{2}{*}{$97.5 \%$} & \multirow{2}{*}{$77.8 \%$} & \multirow{2}{*}{$56.8 \%$} \\
\hline Negative & 139 & 60 & & & & \\
\hline \multicolumn{7}{|l|}{ TNM staging } \\
\hline $0-\mathrm{I}$ & 41 & 13 & \multirow{2}{*}{$80.6 \%$} & \multirow{2}{*}{$34.7 \%$} & \multirow{2}{*}{$50.5 \%$} & \multirow{2}{*}{$68.3 \%$} \\
\hline II-IV & 107 & 54 & & & & \\
\hline \multicolumn{7}{|l|}{ Lauren classification } \\
\hline Intestinal type & 82 & 25 & \multirow{2}{*}{$62.7 \%$} & \multirow{2}{*}{$70.4 \%$} & \multirow{2}{*}{$63.6 \%$} & \multirow{2}{*}{$69.5 \%$} \\
\hline Diffuse type & 66 & 42 & & & & \\
\hline
\end{tabular}

TABLE 3: Multivariate analysis of recurrence-free survival in gastric cancer according to clinicopathological variables and DLEC1 methylation.

\begin{tabular}{llcc}
\hline Clinicopathological variables & HR & $95 \%$ CI & $P$ value \\
\hline Age $(\leq 65$ versus $>65)$ & 1.12 & $0.91-2.04$ & 0.073 \\
Gender (male versus $>$ female) & 1.05 & $0.70-1.21$ & 0.103 \\
Tumor size (<4 versus $\geq 4)$ & 1.58 & $1.16-2.26$ & 0.058 \\
Depth of invasion (Tis-1 versus T2-4) & 3.81 & $1.68-6.85$ & 0.008 \\
Lymphatic invasion (+ versus -) & 4.54 & $2.06-7.36$ & 0.001 \\
Venous invasion (+ versus - ) & 2.86 & $1.43-5.01$ & 0.017 \\
TNM staging (0-I versus II-IV) & 2.32 & $1.26-4.83$ & 0.036 \\
Lauren classification (intestinal versus diffuse type) & 3.66 & $1.37-8.94$ & 0.009 \\
Lymph node metastasis (+ versus -$)$ & 3.92 & $1.78-7.15$ & 0.002 \\
DLEC1 methylation $(>35.10$ versus $\leq 35.10)$ & 2.43 & $1.38-5.07$ \\
\hline
\end{tabular}

\section{Discussion}

CpG islands are DNA segments, at least $0.5 \mathrm{~kb}$ in size, rich in $\mathrm{G}: \mathrm{C}$ and $\mathrm{CpG}$ content, and often located in the promoter or 50-exon sequences of genes. Promoter CpG islands have traditionally been thought to be unmethylated in normal cells. Although the cause is unclear, promoter CpG island hypermethylation can occur in association with cancer development or aging. Promoter CpG island hypermethylation is biologically important for gene function and thought to act as an alternative to genetic change for inactivation of tumor suppressor genes in human gastric cancer [15].

The DLEC1 gene firstly is deleted in lung cancer and located in the 3p22.3 region, which has been identified as one of the common deleted regions in lung cancer [19]. DLEC1 gene encodes a protein which has no significant homology to known proteins or domains and the function of which remains unknown [20]. Functional analyses strongly suggest that DLEC1 is a tumor suppressor gene [21]. Previous research demonstrated the loss of DLEC1 expression in ovarian cancer and the suppression of ovarian cancer cell growth by DLEC1 reexpression. The loss of DLEC1 expression in ovarian cancer is related to promoter hypermethylation and histone hypoacetylation but not to loss of chromosome 3p22.3 [14]. Many studies showed that methylation of the $D L E C 1$ gene correlates with poor prognosis in lung cancer and ovarian cancer [22-24].

In this study, we determined methylation of DLEC1 promoter by quantitative methylation-specific PCR and demonstrated that DLEC1 promoter was hypermethylated in Chinese gastric cancer patients. However, we did not find any correlations between DLEC1 methylation and clinicopathological variables in Chinese gastric cancer patients. Our investigation was similar to other previous studies $[16,23]$. 


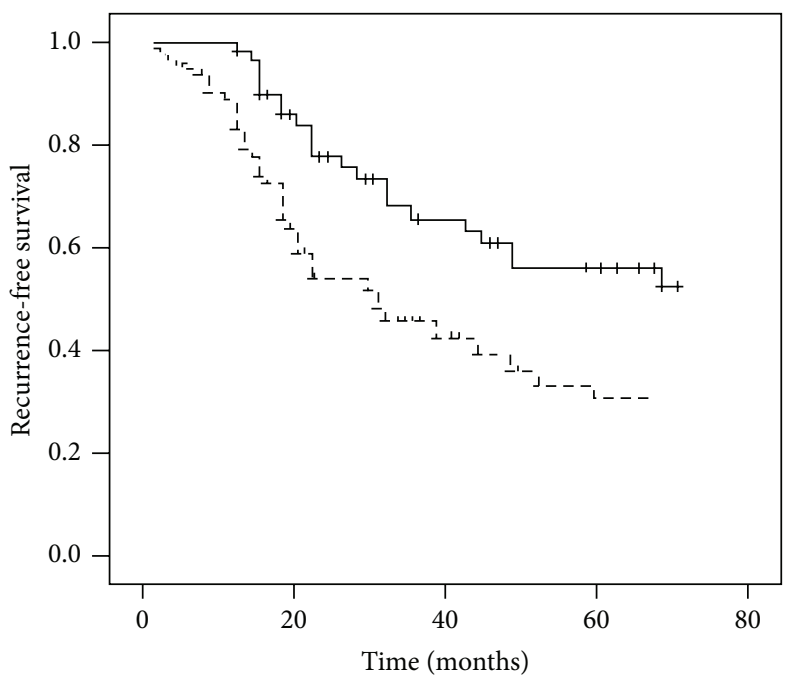

$\begin{array}{rll}r & \text { High DLEC1 methylation } \\ -1 & \text { Low DLEC1 methylation }\end{array}$

FIgURE 3: The recurrence-free survival curves based on DLEC1 methylation. Patients with high DLEC1 methylation had a significantly higher recurrence rate than those with low DLEC1 methylation $(P=0.025)$.

Tumour-specific promoter methylation can serve as a biomarker for prognosis of tumor $[25,26]$. We had found that DLEC1 methylation was significantly higher in patients with recurrence, as compared with that in patients without recurrence among patients without lymph node metastasis. ROC analyses demonstrated that DLEC1 methylation had sensitivity and specificity of $70.1 \%$ and $51.9 \%$, respectively, for recurrence (ROC AUC $=0.648 ; 95 \% \mathrm{CI}, 0.560-0.736$ ). In our study, a multivariate Cox proportional hazards model indicated that DLEC1 methylation was an independent risk factor for recurrence in gastric cancer. Thus, methylation of $D L E C 1$ may be a valuable indicator for recurrence in gastric cancer.

There are some limitations in our study. First, our study population was relatively small and from a single center. Second, we had not measured DLEC1 RNA expression and DLEC1 protein expression in tissues of gastric cancer. However, it would be worthy further exploring the possible use of DLEC1 methylation as a predictor for recurrence in gastric cancer. The multicentric and large-scale prospective validation studies are required in order to confirm our present findings.

\section{Conclusions}

In conclusion, methylation of DELC1 promotermay be a valuable predictor for recurrence in gastric cancer patients.

\section{Conflict of Interests}

The authors declare that there is no conflict of interests regarding the publication of this paper.

\section{Acknowledgment}

This research was supported by Foundation for Young and Middle-Aged Scientists Research of Wannan Medical College (WK201033F).

\section{References}

[1] Ü. Koç, E. Çetinkaya, E. B. Bostanci et al., "Diagnostic significance of serum eotaxin-1 level in gastric cancer patients," Disease Markers, vol. 35, no. 5, pp. 363-367, 2013.

[2] Y. Li, J. Cui, C.-H. Zhang et al., "High-expression of DJ-1 and loss of PTEN associated with tumor metastasis and correlated with poor prognosis of gastric carcinoma," International Journal of Medical Sciences, vol. 10, no. 12, pp. 1689-1697, 2013.

[3] D. E. Guggenheim and M. A. Shah, "Gastric cancer epidemiology and risk factors," Journal of Surgical Oncology, vol. 107, no. 3, pp. 230-236, 2013.

[4] T. Sano, M. Sasako, S. Yamamoto et al., "Gastric cancer surgery: morbidity and mortality results from a prospective randomized controlled trial comparing D2 and extended paraaortic lymphadenectomy-Japan Clinical Oncology Group study 9501," Journal of Clinical Oncology, vol. 22, no. 14, pp. 2767-2773, 2004.

[5] K. W. Kim, B. I. Choi, J. K. Han et al., "Postoperative anatomic and pathologic findings at CT following gastrectomy," Radiographics, vol. 22, no. 2, pp. 323-336, 2002.

[6] J. Whiting, T. Sano, M. Saka, T. Fukagawa, H. Katai, and M. Sasako, "Follow-up of gastric cancer: a review," Gastric Cancer, vol. 9, no. 2, pp. 74-81, 2006.

[7] L. Sun, X.-H. Su, Y.-S. Guan et al., "Clinical role of 18Ffluorodeoxyglucose positron emission tomography/computed tomography in post-operative follow up of gastric cancer: initial results," World Journal of Gastroenterology, vol. 14, no. 29, pp. 4627-4632, 2008.

[8] S. H. Sim, Y. J. Kim, D.-Y. Oh et al., "The role of PET/CT in detection of gastric cancer recurrence," BMC Cancer, vol. 9, article 73, 2009.

[9] M. J. Park, W. J. Lee, H. K. Lim, K. W. Park, J. Y. Choi, and B.-T. Kim, "Detecting recurrence of gastric cancer: the value of FDG PET/CT," Abdominal Imaging, vol. 34, no. 4, pp. 441-447, 2009.

[10] J. F. Costello, M. C. Frühwald, D. J. Smiraglia et al., "Aberrant CpG-island methylation has non-random and tumour-typespecific patterns," Nature Genetics, vol. 24, no. 2, pp. 132-138, 2000.

[11] M. Esteller, P. G. Corn, S. B. Baylin, and J. G. Herman, "A gene hypermethylation profile of human cancer," Cancer Research, vol. 61, no. 8, pp. 3225-3229, 2001.

[12] M. Esteller, "CpG island hypermethylation and tumor suppressor genes: a booming present, a brighter future," Oncogene, vol. 21, no. 35, pp. 5427-5440, 2002.

[13] S. Yamashita, Y. Tsujino, K. Moriguchi, M. Tatematsu, and T. Ushijima, "Chemical genomic screening for methylationsilenced genes in gastric cancer cell lines using 5-aza-2/deoxycytidine treatment and oligonucleotide microarray," Cancer Science, vol. 97, no. 1, pp. 64-71, 2006.

[14] J. Kwong, J.-Y. Lee, K.-K. Wong et al., "Candidate tumorsuppressor gene DLEC1 is frequently downregulated by promoter hypermethylation and histone hypoacetylation in human epithelial ovarian cancer," Neoplasia, vol. 8, no. 4, pp. 268-278, 2006. 
[15] G. H. Kang, S. Lee, N.-Y. Cho et al., "DNA methylation profiles of gastric carcinoma characterized by quantitative DNA methylation analysis," Laboratory Investigation, vol. 88, no. 2, pp. 161-170, 2008.

[16] J. Ying, F. F. Poon, J. Yu et al., “DLEC1 is a functional 3p22.3 tumour suppressor silenced by promoter CpG methylation in colon and gastric cancers," British Journal of Cancer, vol. 100, no. 4, pp. 663-669, 2009.

[17] L. Sobin and C. Wittekind, International Union Against Cancer: TNM Classification of Malignant Tumors, Wiley, New York, NY, USA, 2002.

[18] H. Zheng, H. Takahashi, Y. Murai et al., "Pathobiological characteristics of intestinal and diffuse-type gastric carcinoma in Japan: an immunostaining study on the tissue microarray," Journal of Clinical Pathology, vol. 60, no. 3, pp. 273-277, 2007.

[19] S. Ishikawa, M. Kai, M. Tamari et al., "Sequence analysis of a $685-\mathrm{kb}$ genomic region on chromosome 3p22-p21.3 that is homozygously deleted in a lung carcinoma cell line," DNA Research, vol. 4, no. 1, pp. 35-43, 1997.

[20] W. Al Sarakbi, S. Reefy, W. G. Jiang, T. Roberts, R. F. Newbold, and K. Mokbel, "Evidence of a tumour suppressor function for DLEC1 in human breast cancer," Anticancer Research, vol. 30, no. 4, pp. 1079-1082, 2010.

[21] Q. Zhang, J. Ying, J. Li et al., "Aberrant promoter methylation of DLEC1, a critical 3p22 tumor suppressor for renal cell carcinoma, is associated with more advanced tumor stage," The Journal of Urology, vol. 184, no. 2, pp. 731-737, 2010.

[22] T. J. Seng, N. Currey, W. A. Cooper et al., "DLEC1 and MLH1 promoter methylation are associated with poor prognosis in non-small cell lung carcinoma," British Journal of Cancer, vol. 99, no. 2, pp. 375-382, 2008.

[23] H. Sasaki, Y. Hikosaka, and O. Kawano, "Methylation of the DLEC1 gene correlates with poor prognosis in Japanese lung cancer patients," Oncology Letters, vol. 1, no. 2, pp. 283-287, 2010.

[24] C. Montavon, B. S. Gloss, K. Warton et al., "Prognostic and diagnostic significance of DNA methylation patterns in high grade serous ovarian cancer," Gynecologic Oncology, vol. 124, no. 3, pp. 582-588, 2012.

[25] V. L. Costa, R. Henrique, and C. Jerónimo, "Epigenetic markers for molecular detection of prostate cancer," Disease Markers, vol. 23, no. 1-2, pp. 31-41, 2007.

[26] Y. Wang, Y. Long, Y. Xu et al., "Prognostic and predictive value of $\mathrm{CpG}$ island methylator phenotype in patients with locally advanced nonmetastatic sporadic colorectal cancer," Gastroenterology Research and Practice, vol. 2014, Article ID 436985, 7 pages, 2014. 


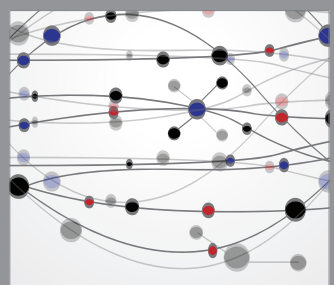

The Scientific World Journal
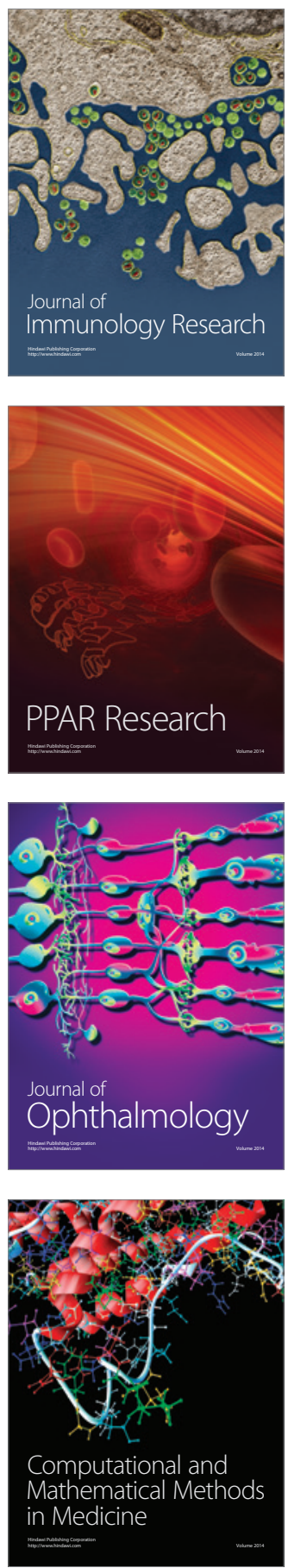

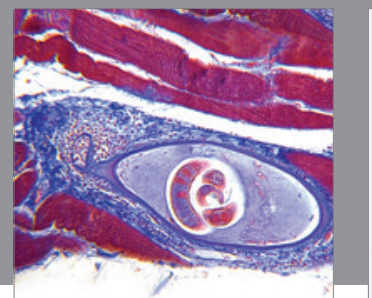

Gastroenterology

Research and Practice
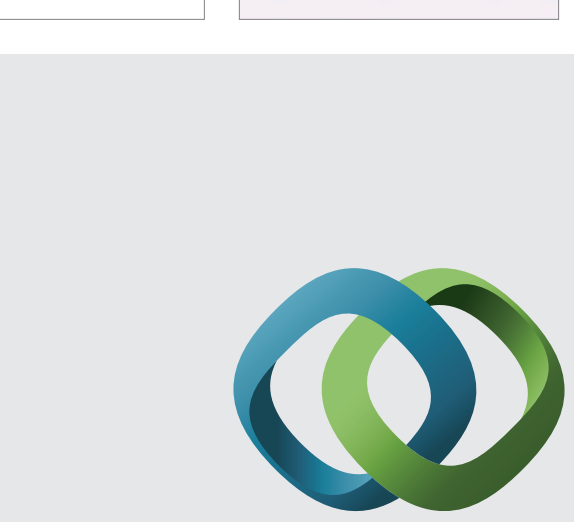

\section{Hindawi}

Submit your manuscripts at

http://www.hindawi.com

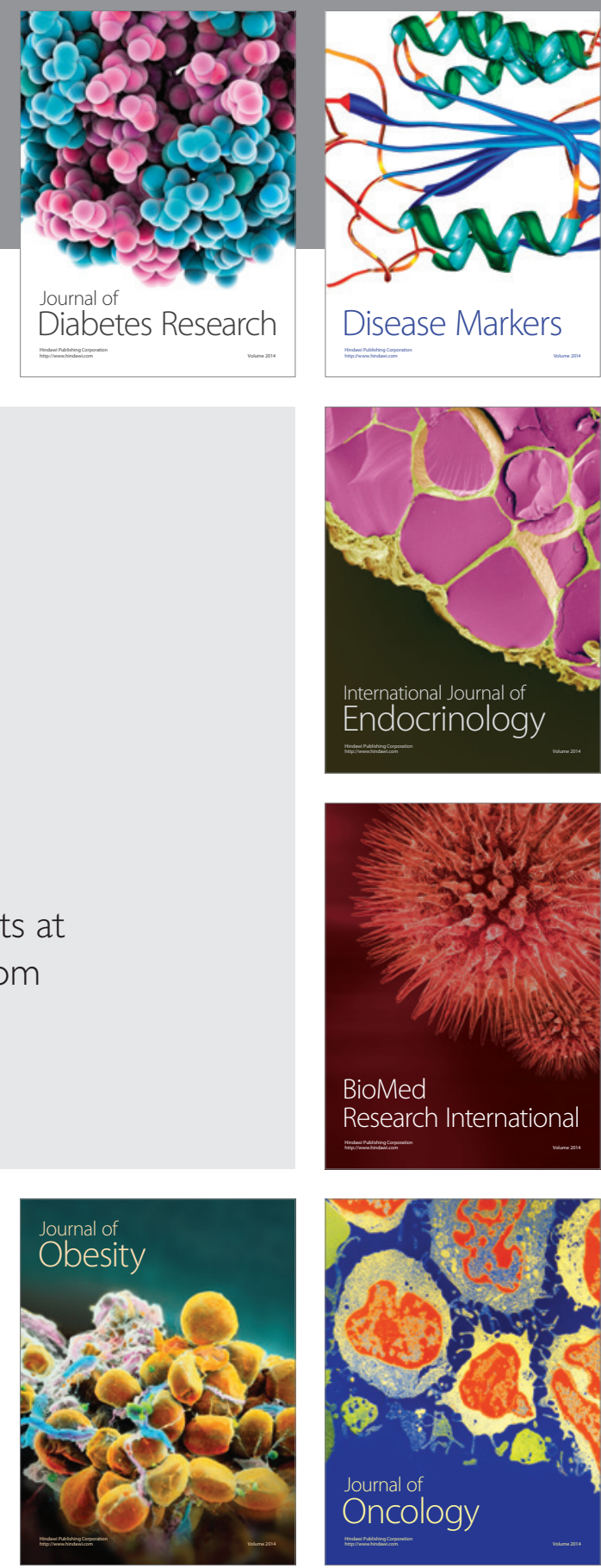

Disease Markers
